\title{
SOME TOPOLOGICAL APPROACHES TO THE FOUR COLOR PROBLEM
}

BY STEVE FISK

\author{
Communicated by Gian-Carlo Rota, March 15, 1973
}

The aim of this paper is to give a few observations with no proofs about the four color problem that are topological in nature. The approach is to define a 'local' 4 coloring on any 2 -manifold and to measure the obstructions to a local coloring's being a desired type of coloring. All manifolds will be assumed to be oriented.

1. Local colorings. We begin with the definitions of four types of coloring that are possible on any surface.

(1) A four coloring of a triangulation $K$ is a map $f: K \rightarrow \partial \Delta^{3}$ which is simplicial, and maps triangles onto triangles. Here $\partial \Delta^{3}$ is the tetrahedron, which has exactly 4 vertices.

(2) An edge coloring of $K$ is a division of the edges of $K$ into 3 sets, so that every triangle of $K$ has an edge in each set. If we think of the sets as colors, every triangle has its edges 3 different colors.

(3) A heawood coloring is an assignment of +1 or -1 to every triangle of $K$ so that the sum of the values on the triangles containing a point $p$ is zero mod 3 for all vertices $p$ of $K$.

(4) A local coloring is a collection of maps $f_{p}: s t(p) \rightarrow \partial \Delta^{3}$ and automorphisms $\sigma_{p q}$ of $\partial \Delta^{3}$ so that the following commutes.

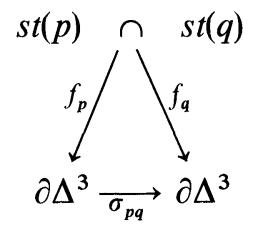

In this definition, $s t(p)$ is the complex consisting of all triangles (and faces of them) containing $p . \sigma_{p q}$ can be given as a permutation of the 4 vertices of $\partial \Delta^{3}$. We can restate the definition by saying we have a 4 coloring for each $s t(p)$ and, up to permutation of colors, the colorings of $s t(p)$ and $s t(q)$ agree on their overlap.

Next, we show how each type of 'coloring' induces the following one. Let $f: K \rightarrow \partial \Delta^{3}$ be a four coloring. $\partial \Delta^{3}$ has an edge coloring, so we get an edge coloring for $K$ by pulling back the one on $\partial \Delta^{3}$. That is, the color of

AMS (MOS) subject classifications (1970). Primary 55A15; Secondary 05C15, 05C10. 
edge $e$ is that of $f(e)$.

If we have an edge coloring, we pick a definite order for the colors, say $a b c$. If the 3 colors of a triangle, going in the positive direction are $a b c$, we assign the triangle +1 . If they are $a c b$, we assign -1 . This turns out to be a heawood coloring.

Suppose we have a heawood coloring. It is not hard to show that, on $s t(p)$, the heawood coloring is induced by a 4 coloring. The collection of these 4 colorings (and appropriate $\sigma_{p q}$ ) gives a local coloring.

In the case of the sphere (or any simply connected region) all of these ways of coloring are equivalent. However, it is easy to find edge colorings of the torus which are not induced by any 4 coloring, etc. To describe this phenomena, we see that if $\alpha$ is a local coloring, then there is a map

$$
\psi(\alpha): \Pi_{1}(M) \rightarrow S(4) .
$$

$S(4)$ is the symmetric group on 4 letters, viewed as the automorphism group of the tetrahedron. One way to see this map is to view the definition of local coloring as defining a fiber bundle with structure group $S(4)$. $\psi(\alpha)$ is then the characteristic map. We have the following

THEOREM 1. Let $\alpha$ be a local coloring of $M$. Then

$\alpha$ is induced by a 4 coloring iff $\operatorname{Im} \psi(\alpha)=(1)$,

$\alpha$ is induced by an edge coloring iff $\operatorname{Im} \psi(\alpha) \subseteq Z_{2} \oplus Z_{2}$,

$\alpha$ is induced by a heawood coloring iff $\operatorname{Im} \psi(\alpha) \subseteq A(4)$.

The groups appearing are all the normal subgroups of $S(4)$. The correspondence between type of coloring and subgroup is given, for example, by $Z_{2} \oplus Z_{2}=$ subgroup of $S(4)$ which preserves a fixed edge coloring of $\partial \Delta^{3}$.

If we have a local coloring $\alpha$, and wonder if it is induced by a heawood coloring, we just check the image of the map

$$
\psi(\alpha)^{\prime}: \Pi_{1}(M) \rightarrow S(4) / A(4) \approx Z_{2} .
$$

Similarly, to see if a heawood coloring is induced by an edge coloring, we have a map

$$
\psi(\alpha)^{\prime \prime}: \Pi_{1}(M) \rightarrow A(4) / Z_{2} \oplus Z_{2} \approx Z_{3} .
$$

2. Conjectures. It is well known that one cannot color an arbitrary 2-manifold with 4 colors. For instance, there are triangulations of the torus that require 7 colors. Our approach is not to increase the number of colors but to change the type of coloring. Thus we formulate the

Generalized 4 Color Conjecture (G4CC). Every triangulation of an orientable 2-manifold has an edge coloring.

If one allows nonorientable surfaces, there is a counterexample. Take 
the icosahedron, and identify antipodal points. The resulting triangulation of the projective plane has no edge coloring, heawood coloring, or even local coloring. The dual of this triangulation is known as the Peterson graph.

Recall that the degree of a vertex in a triangulation is the number of triangles containing it. An even surface is one whose interior vertices all have even degree. If $R$ and $S$ are two triangulations, we can form the connected sum, $R \# S$, by removing one triangle from $R$ and one from $S$, and joining the 2 surfaces so obtained along the boundaries of the triangles.

THEOREM 2. $R$ \# $S$ has an edge coloring iff both $R$ and $S$ have one.

We can construct on the torus a special triangulation $X$ with the property that there are only 2 vertices of $X$ whose degree is odd, and those 2 vertices lie on the same triangle. Using this triangulation $X$ and Theorem 2, we can prove

THEOREM 3. If every even surface has an edge coloring, then G4CC is true.

3. Other descriptions of local colorings. Suppose $\alpha$ is a local coloring of $K$. Let $e$ be an edge of $K$, and $p$ a vertex of $e$. Under the map $f_{p}$, the two triangles containing $e$ either map to the same or different triangles. If they map to different triangles, call $e$ nonsingular. Otherwise call $e$ singular. The concept of singularity and nonsingularity is well defined, and provides a convenient way of representing local colorings. If we label a nonsingular edge with 1 , and a singular edge with 0 , we can ask for ways of telling if a cycle of 0 's and 1's could be the cycle around a vertex in a local coloring. For instance, 1, 1, 0, 0 could not be. There are some straightforward algorithms. The next result is not obvious however:

THEOREM 4. Let $S$ be a cycle of 0's and 1's. Then $S$ could be the cycle of singular and nonsingular edges around a vertex of a local coloring iff there is a triangulation $K$ of a disk $M$ such that

(1) $K$ is even,

(2) the cycle of degrees mod 2 of $\partial K$ is $S$.

4. Stieffel Whitney class of a local coloring. Suppose $\alpha$ is a local coloring. We put $s w(\alpha)$, the Stieffel-Whitney class of $\alpha$, to be the $Z_{2}$ chain of all the singular edges. If $M$ is an even triangulation, there is a canonical local coloring given by having every edge singular. If $M$ is the barycentric subdivision of some other triangulation and hence even, then the StieffelWhitney class of this special local coloring is just the topological StieffelWhitney class. 
It is a fact that $s w(\alpha)$ is a cycle, and so represents a class of $H_{1}\left(M ; Z_{2}\right)$. The next result shows how this class measures the obstruction to $\alpha$ 's being a heawood coloring.

TheOREM 5. Let $\alpha$ be a local coloring of $M$. Define $\overline{\psi(\alpha)}$ by

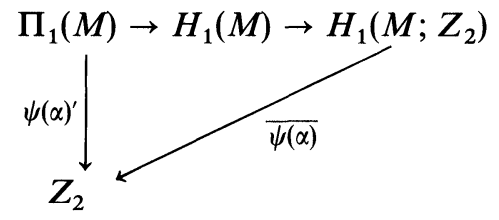

Then for any $X \in H_{1}\left(M ; Z_{2}\right), s w(\alpha) \cap X=\overline{\psi(\alpha)}[X]$.

COROLlary. $\alpha$ is induced by a heawood coloring iff $s w(\alpha)=0$ in $H_{1}\left(M ; Z_{2}\right)$.

We would like to have a similar result when a heawood coloring is induced by an edge coloring.

5. Kempe cycles and color cycles. Suppose $K$ is a triangulation, and $\alpha$ and $\beta$ are two local colorings. Let $\alpha+\beta$ be the chain which is the $Z_{2}$ sum of nonsingular edges of $\alpha$ and $\beta$. Such a chain is actually a $Z_{2}$ cycle, and is called a color cycle of $\alpha$ (or $\beta$ ). Another way to see this is that, if $\gamma$ is a color cycle of $\alpha$ and we change all nonsingular edges of $K$ lying in $\gamma$ to singular, and vice versa, we get another local coloring.

Definition. A Kempe cycle $\gamma$ of a local coloring $\alpha$ is

(1) a $Z_{2}$ cycle,

(2) locally 2 colored. That is, for any $p, f(s t(p) \cap \gamma)$ contains at most 2 vertices.

THEOREM 6. A Kempe cycle of $\alpha$ is a color cycle of $\alpha$.

If we start with a local coloring, and get a new local coloring $\beta$ by changing the edges of a Kempe cycle $\gamma$, we say $\alpha$ is related to $\beta$. Extending this relation to an equivalence relation, we get Kempe equivalence classes of local colorings. For the sphere, this is the same as the usual definition.

REMARK. Suppose we change a local coloring $\alpha$ along a color cycle $\gamma$, obtaining a local coloring $\beta$. Then $\operatorname{sw}(\alpha)+\gamma=\operatorname{sw}(\beta)$.

This remark, combined with the corollary, shows us that if we start with a local coloring $\alpha$ of $M$ and can find a color cycle $\gamma$ such that $s w(\alpha)=\gamma$ in $H_{1}\left(M ; Z_{2}\right)$, then $M$ has a heawood coloring.

Question. For a fixed local coloring $\alpha$ of $M$, which classes of $H_{1}\left(M ; Z_{2}\right)$ are represented by

(1) Kempe cycles,

(2) color cycles. 
We cannot answer these, but if we slightly weaken the conditions we get some partial results. Let an assignment of singular and nonsingular edges of $M$ be called a local coloring with a singularity if, at all points except perhaps one, there are maps $f_{p}$ as in the definition. A Kempe cycle with singularity of a local coloring is a $Z_{2}$ chain which is locally 2 colored at all but perhaps one point. A color cycle with singularity is the sum of a local coloring and a local coloring with singularity. There are no singular objects on any simply connected region.

THEOREM 7. If $\alpha$ is a local coloring, $\gamma$ a color cycle with singularity, and $s w(\alpha)=\gamma$ in $H_{1}\left(M ; Z_{2}\right)$ then if we change $\alpha$ along $\gamma$, we get a heawood coloring.

Definition. $M$ is sufficiently fine if there is a vertex $p$ of $M$ and paths of triangles containing $p$ such that

(1) any two paths of triangles meet only in $p$,

(2) the paths contain generators of $H_{1}(M)$.

For a given triangulation, some iterated barycentric subdivision is sufficiently fine.

THEOREM 8. If $M$ is a triangulation such that

(1) $M$ is sufficiently fine,

(2) $M$ is even,

then $M$ has a heawood coloring.

This follows from the fact that every class of $H_{1}\left(M ; Z_{2}\right)$ is represented by a Kempe chain with a singularity.

CONJECTURE. For any local coloring of a triangulation $M$, every class of $H_{1}\left(M ; Z_{2}\right)$ is represented by a Kempe cycle (color cycle) with a singularity.

6. Trying to prove the 4 color conjecture. We end this paper with an outline of how to prove the 4-color conjecture (generalized).

(1) Remove the topological restrictions in Theorem 8 or decide the last conjecture.

(2) Find a class in $H_{1}\left(M ; Z_{3}\right)$ measuring the obstruction to a heawood coloring's being an edge coloring.

(3) From what was learned in solving 1, show how to 'deform' a heawood coloring to an edge coloring.

Department of Mathematics, Massachusetts Institute of Technology, Cambridge, MASSACHUSETTS 02139 